\title{
Electronic structure evolutions driven by oxygen vacancy in $\mathrm{SrCoO}_{3-x}$ films
}

Jiali Zhao ${ }^{1,5}$, Yi Luo ${ }^{2}$, Jia-Ou Wang ${ }^{1}$, Haijie Qian ${ }^{1}$, Chen Liu ${ }^{1}, \mathrm{Xu} \mathrm{He}^{3}$, Qinghua Zhang ${ }^{4,5}$, Heyi Huang ${ }^{4,5}$, Bingbing Zhang ${ }^{1}$, Shunfang $\mathrm{Li}^{2}$, Erjia Guo ${ }^{4}$, Chen Ge ${ }^{4}$, Tieying Yang ${ }^{6}$, Xiaolong $\mathrm{Li}^{6}$, Meng He${ }^{4}$, Lin Gu ${ }^{4,5}$, Kui-Juan Jin ${ }^{4,5}$, Kurash Ibrahim ${ }^{1^{*}}$ and Haizhong Guo ${ }^{2^{*}}$

\begin{abstract}
Ionic defects, such as oxygen vacancies, play a crucial role in the magnetic and electronic states of transition metal oxides. Control of oxygen vacancy is beneficial to the technological applications, such as catalysis and energy conversion. Here, we investigate the electronic structure of $\mathrm{SrCoO}_{3-x}$ as a function of oxygen content $(x)$. We found that the hybridization extent between Co $3 \mathrm{~d}$ and $\mathrm{O} 2 \mathrm{p}$ increased with the reduction of oxygen vacancies. The valence band maximum of $\mathrm{SrCoO}_{2.5+\delta}$ has a typical $\mathrm{O} 2 \mathrm{p}$ characteristic. With further increasing oxygen content, the Co ions transform from a high spin $\mathrm{Co}^{3+}$ to an intermediate spin $\mathrm{Co}^{4+}$, resulting in a transition of $\mathrm{SrCoO}_{3-x}$ from insulator to metal. Our results on the electronic structure evolution with the oxygen vacancies in $\mathrm{SrCoO}_{3-x}$ not only illustrate a spin state transition of Co ions, but also indicate a perspective application in catalysis and energy field.
\end{abstract}

Keywords: oxygen vacancies, $\mathrm{SrCoO}_{3-x}$, electronic structure evolution, resonant photoemission spectra

\section{INTRODUCTION}

The characteristics of transition metal oxides (TMOs) endow them with various applications in the fields of the cutting-edge research, such as energy storage and generating devices $[1,2]$. Oxygen vacancy has flexible degree of freedom to manipulate the functionalities of TMOs [3] due to its strong influence on the number of electrons in the $3 \mathrm{~d}$ orbital of transition metals. In the cobaltites, oxygen vacancy could modify both valence and spin state of Co ions, and thus effectively influence the physical properties $[4,5]$. $\mathrm{SrCoO}_{3-x}$ can be stabilized in two reversible topotactic phases, i.e., $\mathrm{SrCoO}_{2.5}$ and $\mathrm{SrCoO}_{3}$, by simply changing the oxygen content using in-situ or exsitu exterior methods [5-10]. The physical properties of these two materials are in sharp contrast. $\mathrm{SrCoO}_{2.5}$ is an antiferromagnetic insulator with alternating $\mathrm{CoO}_{4}$ tetrahedral and $\mathrm{CoO}_{6}$ octahedral sub-layers, whereas $\mathrm{SrCoO}_{3}$ is a ferromagnetic metal composed of $\mathrm{CoO}_{6}$ octahedral. Due to a very small difference of Gibbs free energy between $\mathrm{SrCoO}_{2.5}$ and $\mathrm{SrCoO}_{3}$, they can be easily transformed from one phase to the other at relatively low temperature [11-14]. Previous work had demonstrated that the reversible topotactic phase transformation can be achieved by either physical or chemical methods. In physical methods, the oxygen vacancies in $\mathrm{SrCoO}_{3-x}$ can be tuned by annealing in high oxygen atmosphere or vacuum environment. The chemical approaches include electrolysis and direct treatment with oxidizing or reducing agents. Jeen et al. [7] reported phase transformation from $\mathrm{SrCoO}_{3-\delta}$ to $\mathrm{SrCoO}_{2.5}$ by annealing the sample at $\sim 210^{\circ} \mathrm{C}$ in vacuum. The phase transformation is reversible as $\mathrm{SrCoO}_{2.5}$ can transform into $\mathrm{SrCoO}_{3-\delta}$ by annealing at $\sim 350^{\circ} \mathrm{C}$ in pure oxygen. Recently, Lu et al. [13] illustrated the tri-state transition among the $\mathrm{SrCoO}_{3-\delta}$, $\mathrm{SrCoO}_{2.5}$, and $\mathrm{HSrCoO}_{2.5}$ using electric-field driven ionic transport. Besides, $\mathrm{SrCoO}_{2.5}$ is successfully oxidized to $\mathrm{SrCoO}_{3}$ by putting the as-deposited $\mathrm{SrCoO}_{2.5}$ thin films in dilute $\mathrm{NaClO}$ solutions [11].

During the topotactic phase transformation, oxygen vacancy content is the only variable to trigger Co ions

\footnotetext{
${ }^{1}$ Institute of High Energy Physics, Chinese Academy of Sciences, Beijing 100049, China

${ }^{2}$ School of Physical Engineering, Zhengzhou University, Zhengzhou 450001, China

${ }^{3}$ Physique Théorique des Matériaux, Q-MAT, CESAM, Université de Liège, B-4000 Liège, Belgium

${ }^{4}$ Institute of Physics, Chinese Academy of Sciences, Beijing 100190, China

${ }^{5}$ University of Chinese Academy of Sciences, Beijing 100049, China

${ }^{6}$ Shanghai Synchrotron Radiation Facility (SSRF), Shanghai Institute of Applied Physics, Chinese Academy of Sciences, Shanghai 201204, China

* Corresponding authors (emails: kurash@ihep.ac.cn (Ibrahim K); hguo@zzu.edu.cn (Guo H))
} 
changing from high-spin state $\mathrm{Co}^{3+}$ ions in $\mathrm{SrCoO}_{2.5}$ to intermediate-spin state $\mathrm{Co}^{4+}$ ions in $\mathrm{SrCoO}_{3}$. The physical properties will change with the spin state and valence of Co ions accordingly [15]. In order to find out the underlying mechanism controlling the physical properties of $\mathrm{SrCoO}_{2.5}$ and $\mathrm{SrCoO}_{3}$, one needs to understand their electronic structures. However, an unambiguous experimental observation of electronic structure changes induced by oxygen vacancies in $\mathrm{SrCoO}_{3-x}$ is still lacking.

The amount of the oxygen vacancies of $\mathrm{SrCoO}_{3-x}$ $(0 \leq x \leq 0.5)$ was tuned by strain and chemical oxidation. Afterwards, the electronic structure evolution of $\mathrm{SrCoO}_{3-x}$ driven by variation of the oxygen vacancies was illustrated by a combination of X-ray absorption spectra and resonant photoemission spectra. From $\mathrm{SrCoO}_{2.5}$ to $\mathrm{SrCo}_{2.5+\delta}$, the variation of oxygen content would strengthen the hybridization between Co $3 \mathrm{~d}$ and $\mathrm{O} 2 \mathrm{p}$ with the valence band maximum mainly having $\mathrm{O} 2 \mathrm{p}$ characteristic, resulting in the transformation from high spin $\mathrm{Co}^{3+}$ in $\mathrm{SrCo}_{2.5+\delta}$ to intermediate spin $\mathrm{Co}^{4+}$ in $\mathrm{SrCoO}_{3-\delta}$, and thus the transformation from insulator to metal.

\section{EXPERIMENTAL SECTION}

$\mathrm{SrCoO}_{2.5}$ thin films (24 nm-thick and $187 \mathrm{~nm}$-thick) were epitaxially grown on (001)-oriented $\mathrm{LaAlO}_{3}$ (LAO) substrates by pulsed laser deposition (PLD). All films were deposited at $700^{\circ} \mathrm{C}$ using an excimer $\mathrm{XeCl}$ laser $\left(1.5 \mathrm{~J} \mathrm{~cm}^{-2}, 308 \mathrm{~nm}, 2 \mathrm{~Hz}\right)$ at oxygen partial pressure of $10 \mathrm{~Pa}$. After deposition, the films were in situ annealed for $30 \mathrm{~min}$ and then cooled down to room temperature. The growth details can be found in our previous work [16]. The $24 \mathrm{~nm}$-thick $\mathrm{SrCoO}_{2.5}$ film was coherently strained to the substrate, whereas the $187 \mathrm{~nm}$-thick film was partially strain relaxed. For comparison, the $24 \mathrm{~nm}$-thick $\mathrm{SrCoO}_{2.5}$ thin film was cut into two parts. One was immersed into $\mathrm{NaClO}$ solution at room temperature for $20 \mathrm{~min}$, denoted as oxidized-SrCoO $3-x$, whereas the other was kept in the as-grown state.

The crystal structure of the as-prepared brownmillerite-

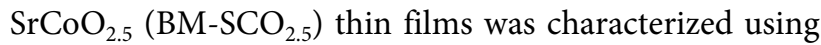
an ARM-200CF (JEOL, Tokyo, Japan), state-of-the-art spherical aberration-corrected scanning transmission electron microscopy (STEM) techniques of the high-angle annular dark-field (HAADF) and annular bright-field $(\mathrm{ABF})$-operated being equipped with double spherical aberration (Cs) correctors. X-ray diffraction (XRD) was collected with Rigaku SmartLab $(9 \mathrm{~kW})$ High-resolution (Ge 220×2) X-Ray Diffractometer with $1.54 \AA$ X-rays. Xray reciprocal space mapping (RSM) of $\mathrm{SrCoO}_{3-x}$ thin films were performed with high-resolution synchrotron $\mathrm{X}$-ray diffractometry at the BL14B1 beam line of Shanghai Synchrotron Radiation Facility (SSRF) using a $1.24 \AA \mathrm{X}$ rays with a Huber 5021 six-axis diffractometry. Temperature dependences of resistivity were performed using a Physical Properties Measurement System (PPMS, Quantum Design Inc.). X-ray absorption spectra (XAS) and resonant photoemission spectra (RPES) were performed at the 4B9B beam line at Beijing Synchrotron Radiation Facility of Institute of High Energy Physics, Chinese Academy of Sciences (CAS). The ultrahigh vacuum chamber background pressure is about $2 \times 10^{-10}$ Torr. The O-K edge and Co-L edge XAS were recorded in the total-electron-yield (TEY) mode.

\section{RESULTS AND DISCUSSION}

Fig. $1 \mathrm{a}, \mathrm{b}$ show the HAADF-STEM images of $\mathrm{BM}^{-\mathrm{SCO}_{2.5}}$ thin films with thicknesses of 24 and $187 \mathrm{~nm}$, respectively. Both interfaces in these two films are atomically sharp without any evidence of misfit dislocations or chemical reaction in the interface region. All films are coherently strained by the substrate. The STEM result demonstrates the $\mathrm{BM}-\mathrm{SCO}_{2.5}$ thin films are highly epitaxial grown.

ABF-STEM can detect light atoms, such as oxygen [17]. Fig. $1 c$, d show the ABF-STEM images of $\mathrm{BM}^{-\mathrm{SCO}_{2.5}}$ thin films with thicknesses of 24 and $187 \mathrm{~nm}$, respectively. Line profiles along the vertical direction indicated by red and blue lines in ABF-STEM images of Fig. 1c, $d$ are shown in Fig. 1e, f, respectively. The alternate stacking is observed in Fig. 1d, originating from the fully oxygenated octahedral and oxygen-deficient tetrahedral sub-layers. This result is consistent with the previous report [7],

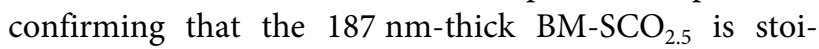
chiometric.

The intensity of oxygen ions in the $24 \mathrm{~nm}$-thick BM$\mathrm{SCO}_{2.5}$ film is stronger than that of the $187 \mathrm{~nm}$-thick BM$\mathrm{SCO}_{2.5}$ film (Fig. 1e, $\mathrm{f}$ ), indicating the oxygen content in the thinner film is more than that of the thicker film. Therefore, we defined the $24 \mathrm{~nm}$-thick and $187 \mathrm{~nm}$-thick $\mathrm{SrCoO}_{3-x}$ film as $\mathrm{SrCoO}_{2.5+\delta}$ and $\mathrm{SrCoO}_{2.5}$ thereafter, respectively.

Fig. 2a shows the XRD $\theta-2 \theta$ scans of $\mathrm{SrCoO}_{2.5}$, $\mathrm{SrCoO}_{2.5+\delta}$ and $24 \mathrm{~nm}$-thick oxidized-SrCoO${ }_{3-x}$. Only $(00 l)$ peaks were observed in the XRD $\theta-2 \theta$ scans, indicating all films are of single phase and $c$-axis-oriented. RSM images are shown in Fig. 3. The $\mathrm{SrCoO}_{3-x}$ thin films are coherently grown on the LAO substrates. Moreover, for the $24 \mathrm{~nm}$-thick oxidized-SrCoO $3-x$ thin film, the peaks originating from the alternatively-stacked octahe- 

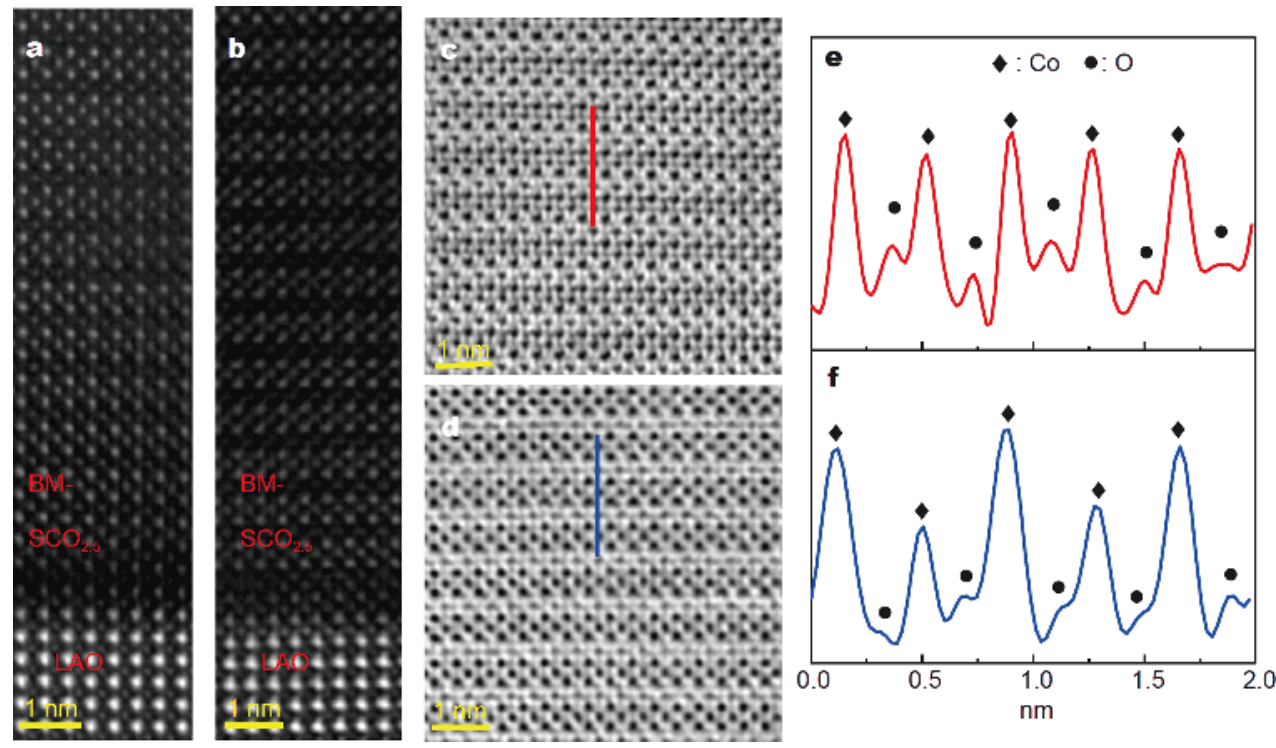

Figure 1 HAADF-STEM images of (a) $24 \mathrm{~nm}$-thick and (b) $187 \mathrm{~nm}$-thick BM-SCO ${ }_{2.5}$ thin films, respectively. ABF-STEM images of (c) $24 \mathrm{~nm}$-thick and (d) $187 \mathrm{~nm}$-thick BM-SCO 2.5 thin films, respectively. (e, f) Line profiles of the inversed ABF contrasts indicated by the red and blue lines in $\mathrm{ABF}$ images (c) and (d), respectively (Lines in (c) and (d) can be red and blue accordingly).

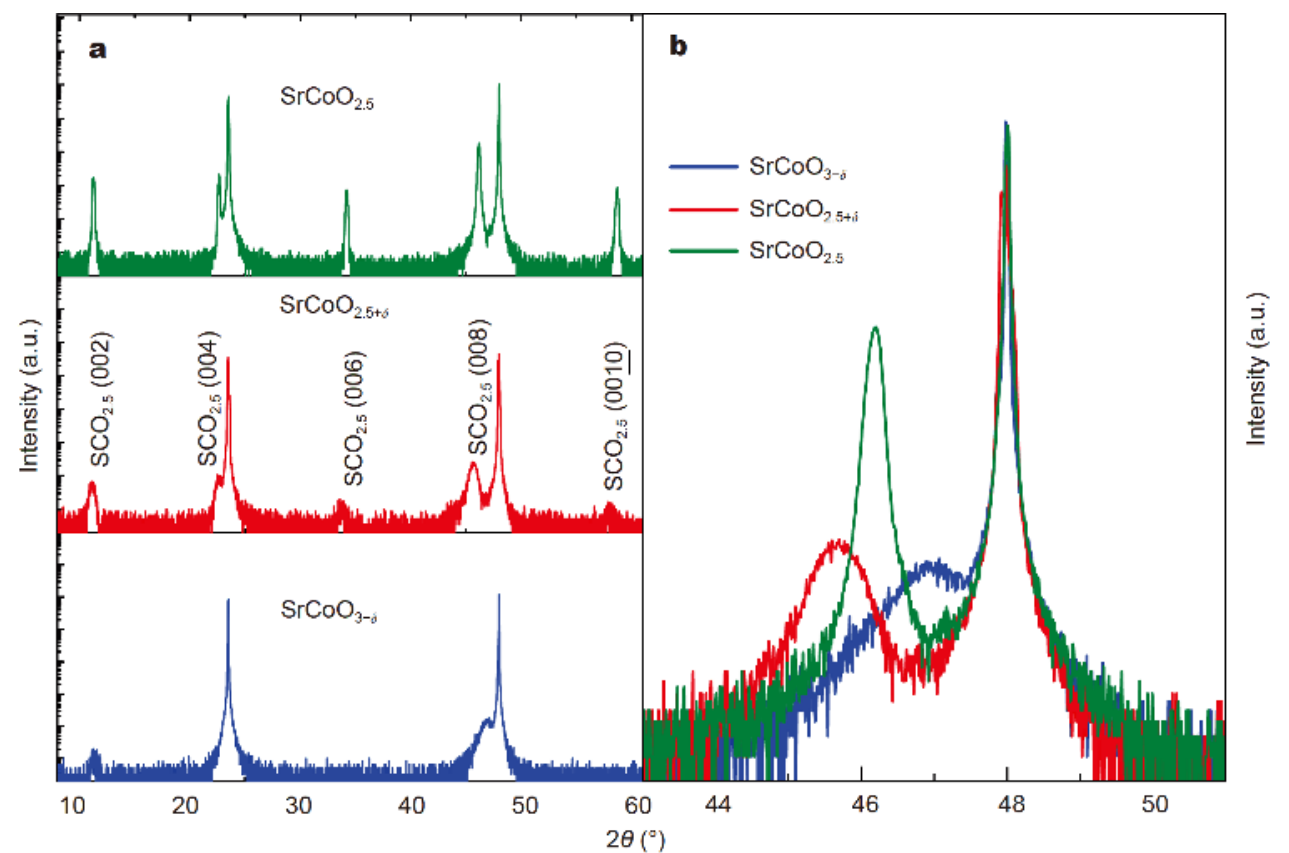

Figure 2 (a) XRD $\theta-2 \theta$ patterns of $\mathrm{SrCoO}_{2.5}, \mathrm{SrCoO}_{2.5+\delta}$, and $\mathrm{SrCoO}_{3-\delta}$. (b) Enlarged part of (a).

dral and tetrahedral sublayers almost disappear, which indicates that the $24 \mathrm{~nm}$-thick oxidized-SrCoO $3-x$ thin film was oxidized in $\mathrm{NaClO}$ solution, and its oxygen content is more than 2.75 and close to 3 , therefore, we defined this sample as $\mathrm{SrCoO}_{3-\delta}$.
The lattice mismatch between LAO $(a=3.792 \AA)$ and $\mathrm{SrCoO}_{2.5}(a=3.905 \AA)$ is about $2.98 \%$. When $\mathrm{SrCoO}_{2.5}$ thin film grew on the LAO substrate, it suffers an in-plane compressive strain. Fig. $2 b$ shows the enlarged part of Fig. 2a. The (008) peak of $\mathrm{SrCoO}_{2.5}$ thin film is located at 

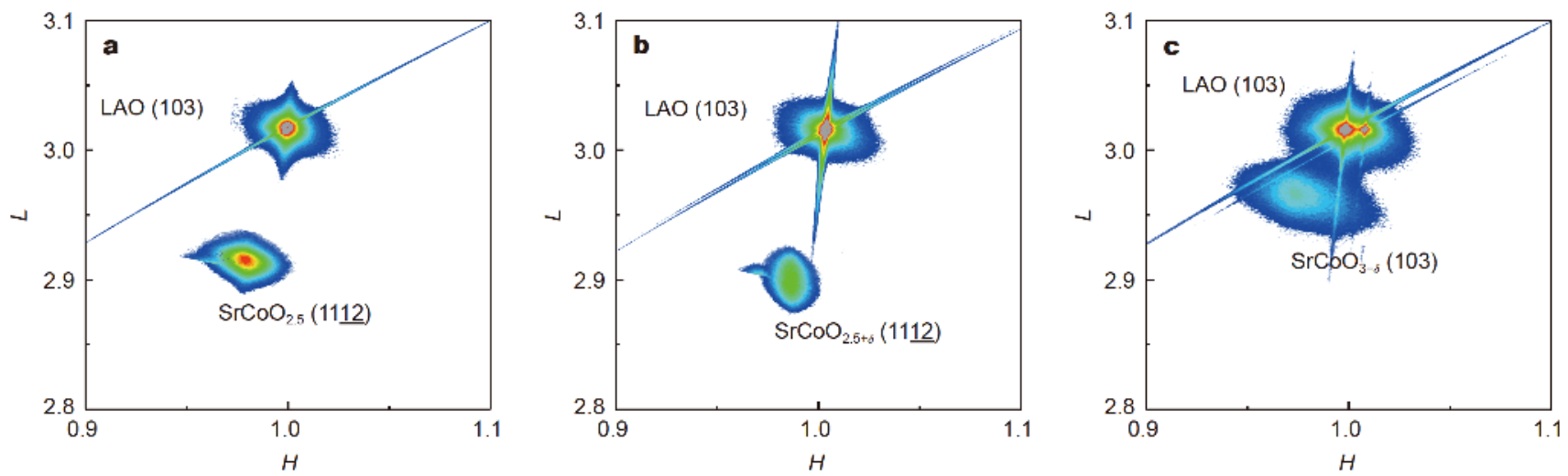

Figure 3 Reciprocal space maps around the LAO (103) Bragg reflection of three $\mathrm{SrCoO}_{3-x}$ films with different oxygen contents: SrCoO ${ }_{2.5}(\mathrm{a})$, $\mathrm{SrCoO}_{2.5+\delta}$ (b), and $\mathrm{SrCoO}_{3-\delta}(\mathrm{c})$.

higher $2 \theta$ angle region than that of $\mathrm{SrCoO}_{2.5+\delta}$ thin film. It indicates that with the increase of the film thickness, the (008) peak shifts towards high $2 \theta$ angle region and the $c^{-}$ axis lattice constant decreases. The in-plane compressive strain of the thin films relaxes with the increase of its thickness. The (002), (006) and (0010) peaks of $\mathrm{SrCoO}_{2.5+\delta}$ film appear, suggesting the alternation of $\mathrm{CoO}_{4}$ tetrahedral layers and $\mathrm{CoO}_{6}$ octahedral layers. Therefore, the oxygen content of $\mathrm{SrCoO}_{2.5+\delta}$ film is no more than 2.75 $(0<\delta<0.25)$. The $(008)$ peak of $\mathrm{SrCoO}_{3-\delta}$ thin film shifts toward higher $2 \theta$ angle region with respect to that of $\mathrm{SrCoO}_{2.5+\delta}$ thin film. The higher the $2 \theta$ angle is, the smaller the $c$-axis constant is. As the thicknesses of these two samples are same, the in-plane compressive strain should be same. The difference of the $c$-axis lattice constants of these two samples is due to the variation of oxygen content. The oxygen vacancies in $\mathrm{SrCoO}_{3-x}$ lead to the expansion of lattice constants. The more the oxygen vacancies exist, the larger the lattice constant is.

In order to further investigate the electronic transport properties of $\mathrm{SrCoO}_{3-x}$ thin films, temperature dependent transport properties were performed by PPMS. Fig. 4 shows the profiles of resistivity versus temperature for $\mathrm{SrCoO}_{2.5}, \mathrm{SrCoO}_{2.5+\delta}$, and $\mathrm{SrCoO}_{3-\delta}$ thin films. $\mathrm{SrCoO}_{2.5}$ film exhibits a highly insulating behavior. A significant reduction of one order of magnitude is in the $\mathrm{SrCoO}_{2.5+\delta}$ thin films. A clear metallic behavior is observed in the $\mathrm{SrCoO}_{3-\delta}$ thin film. An enhancement of resistivity upon the increase of the oxygen vacancies in the $\mathrm{SrCoO}_{3-x}$ films is clearly shown.

Next, XAS was performed to provide the information about the electronic dipole excitation of $2 \mathrm{p}_{1 / 2}\left(\mathrm{~L}_{2}\right)$ and $2 \mathrm{p}_{3 / 2}\left(\mathrm{~L}_{3}\right)$ core electrons to the unoccupied $3 \mathrm{~d}$ bands. The branching ratio of the peak $\left(\mathrm{L}_{3} /\left(\mathrm{L}_{3}+\mathrm{L}_{2}\right)\right)$, the shape of the

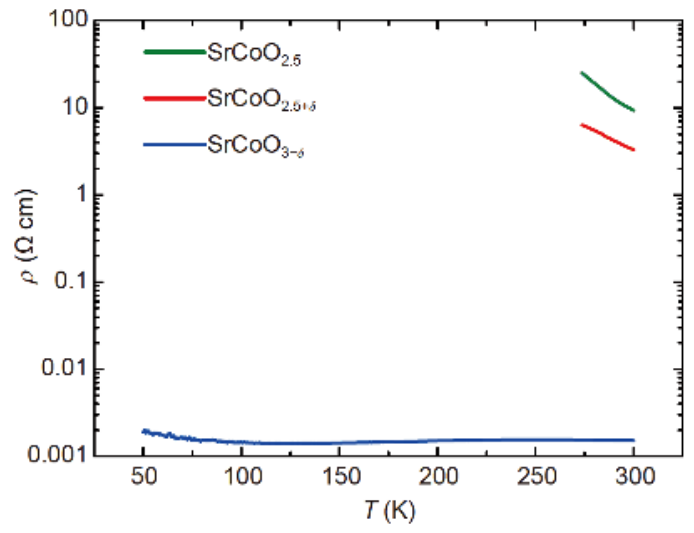

Figure 4 Resistivity versus temperature for $\mathrm{SrCoO}_{2.5}, \mathrm{SrCoO}_{2.5+\delta}$, and $\mathrm{SrCoO}_{3-\delta}$ thin films, showing a clear enhancement of resistivity upon the increase of oxygen vacancies.

peak, and the position of TM L-edge XAS were determined by the electron number and the spin states of the TM $3 \mathrm{~d}$ states. The oxygen vacancies alter the valence state of the Co ions and the hybridization between Co 3d and $\mathrm{O} 2 \mathrm{p}$ in the $\mathrm{SrCoO}_{3-x}$ thin film, therefore the line shape of Co-L edge XAS directly reflects the oxygen contents in the $\mathrm{SrCoO}_{3-x}$ thin films. Fig. 5a shows the Co$\mathrm{L}$ edge XAS of $\mathrm{SrCoO}_{2.5}, \mathrm{SrCoO}_{2.5+\delta}$, and $\mathrm{SrCoO}_{3-\delta}$ thin films. The positions of the $\mathrm{L}_{3}$ peaks of $\mathrm{SrCoO}_{2.5}$, $\mathrm{SrCoO}_{2.5+\delta}$, and $\mathrm{SrCoO}_{3-\delta}$ thin films are located at 780.05, 780.18 , and $780.55 \mathrm{eV}$, respectively. In the Co-L edge XAS, the higher the photon energy of the peak is, the higher the valence state of Co ions is. The peak position of $\mathrm{L}_{3}$-edge shifts towards the higher energy region with the reduction of oxygen vacancies, indicating that the valence of $\mathrm{Co}$ ions increases. $\mathrm{L}_{3} /\left(\mathrm{L}_{3}+\mathrm{L}_{2}\right)$ of $\mathrm{SrCoO}_{2.5}$, $\mathrm{SrCoO}_{2.5+\delta}$, and $\mathrm{SrCoO}_{3-\delta}$ thin films which reflects the 

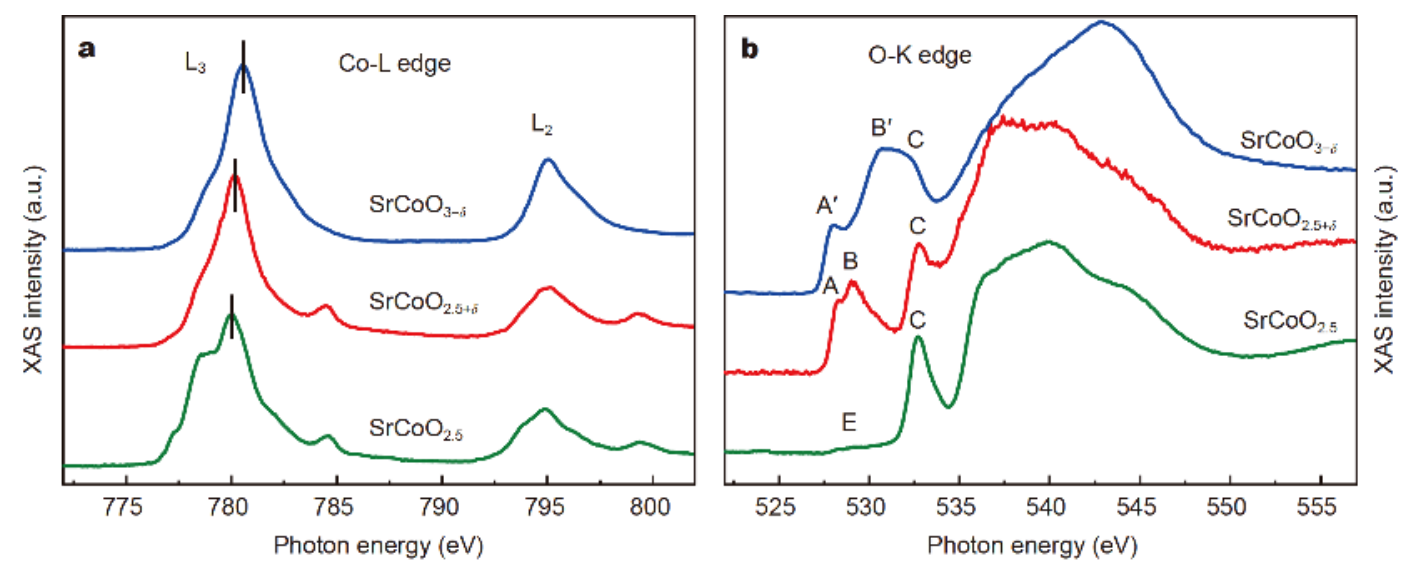

Figure 5 XAS of $\mathrm{SrCoO}_{2.5}, \mathrm{SrCoO}_{2.5+\delta}$, and $\mathrm{SrCoO}_{3-\delta}$ films: (a) Co-L edge and (b) O-K edge.

spin state of Co ions is calculated as $0.75,0.76$, and 0.70 , respectively. From the line shape and the branching ratio of Co-L edge XAS, we can conclude that $\mathrm{Co}$ ions in the $\mathrm{BM}-\mathrm{SCO}_{2.5}$ and $\mathrm{SrCoO}_{2.5+\delta}$ are at high-spin state, while the Co ions in perovskite $\mathrm{SrCoO}_{3-\delta}$ are at intermediatespin state, which is in agreement with the previous calculation and experiments [15,18-21].

$\mathrm{O}-\mathrm{K}$ edge XAS originates from the transition from the $\mathrm{O}$ 1s core-level state to the unoccupied $\mathrm{O} 2 \mathrm{p}$ state. The completely ionic oxides would not show any peaks in the $\mathrm{O}-\mathrm{K}$ edge XAS due to the completely occupied O 2p state. Therefore, the O-K edge XAS provides not only the information about the unoccupied $\mathrm{O} 2 \mathrm{p}$ state, but also the information about the hybridization between Co $3 \mathrm{~d}$ and O 2p. Fig. 5 b shows that the pre-peak $\mathrm{E}$ of $\mathrm{SrCoO}_{2.5}$ is too weak to be detected. The pre-peak $\mathrm{E}$ is attributed to the hybridization of $\mathrm{O} 2 \mathrm{p}$ with Co $3 \mathrm{~d}$ in the octahedral coordination. The peak $\mathrm{C}$ represents the superoxide species $\mathrm{O}_{2}^{-}$that commonly exist in the surface region of the oxygen-deficiency $\mathrm{SrCoO}_{3-x}$ [22]. The intensity of peak $\mathrm{C}$ reduces, meanwhile the pre-peak $\mathrm{A}$ and peak $\mathrm{B}$ appear with the increase of oxygen content. These observations can be attributed to the hybridization of $\mathrm{O} 2 \mathrm{p}$ with $\mathrm{Co} 3 \mathrm{~d}$ $t_{2 g}$ and $e_{g}$ in octahedral coordination, respectively. As the oxygen content increases, peaks A and B shift toward the higher photon energy region, where they are defined as peaks $\mathrm{A}^{\prime}$ and $\mathrm{B}^{\prime}$, respectively. The calculated intensity ratio of peak $A$ and $B$ decreases significantly with the reduction of oxygen vacancies, suggesting that Co ions in $\mathrm{SrCoO}_{3-\delta}$ are at intermediate-spin $4+$ state, whereas $\mathrm{Co}$ ions in $\mathrm{SrCoO}_{2.5+\delta}$ are at high-spin 3+ state. Peaks A and $\mathrm{A}^{\prime}$ represent the unoccupied states of Co $3 \mathrm{~d} \mathrm{t}_{2 \mathrm{~g}}$ orbital of high-spin $\mathrm{Co}^{3+}$ and intermediate-spin $\mathrm{Co}^{4+}$, respectively. Meanwhile, Peaks B and B' represent the unoccupied state of the Co $3 \mathrm{~d} \mathrm{e}_{\mathrm{g}}$ orbital of high-spin $\mathrm{Co}^{3+}$ and intermediate-spin $\mathrm{Co}^{4+}$, respectively. The unoccupied state of the Co $3 \mathrm{~d} \mathrm{t}_{2 \mathrm{~g}}$ orbital of the high-spin $\mathrm{Co}^{3+}$ and the intermediate-spin $\mathrm{Co}^{4+}$ is the same. However, the unoccupied state of Co $3 \mathrm{~d} \mathrm{e}_{\mathrm{g}}$ orbital of the intermediate-spin $\mathrm{Co}^{4+}$ is more than that of the high-spin $\mathrm{Co}^{3+}$. Therefore, the intensity ratio of peak $\mathrm{A}$ and $\mathrm{B}$ of $\mathrm{SrCoO}_{2.5+\delta}$ is much larger than that of $\mathrm{SrCoO}_{3-\delta}$.

To investigate the evolution of the valence band electronic structure with the variation of oxygen vacancies in $\mathrm{SrCoO}_{3-x}$, the Co 2p-3d RPES were measured and shown in Fig. 6. Three energy regions (labeled with cyan, magenta, and green arrows in Fig. 6a) in the Co-L edge XAS were selected to do the resonant photoemission and measure the electronic structure in the valence band, shown in Fig. $6 \mathrm{~b}$ with colors corresponding to the colors of the arrow in Fig. 6a. The selected energy regions cover the off-resonance and on-resonance photon energy in order to compare the on-resonance and off-resonance effects. The on-resonance photon energy enhances the transition from Co $2 \mathrm{p}$ to Co $3 \mathrm{~d}$, and mainly shows the contribution of Co $3 \mathrm{~d}$ in valence band. From the offresonance to on-resonance photon energy, the contribution of Co $3 \mathrm{~d}$ electrons to the valence band electronic structure would be enhanced. For $\mathrm{SrCoO}_{2.5}$, the intensity of the peak around $4 \mathrm{eV}$ below Fermi level is obviously enhanced at the on-resonance photon energy, indicating that the valence band is dominated by the Co $3 \mathrm{~d}$ characteristic. For $\mathrm{SrCoO}_{2.5+\delta}$, the intensity of the peak around $3 \mathrm{eV}$ below Fermi level decreases when the photon energy shifts from the off-resonance region to the on-resonance region. This result is attributed to the valence band maximum dominated by the $\mathrm{O} 2 \mathrm{p}$ characteristic. The O $2 p$ characteristic peak shifts toward the Fermi level 


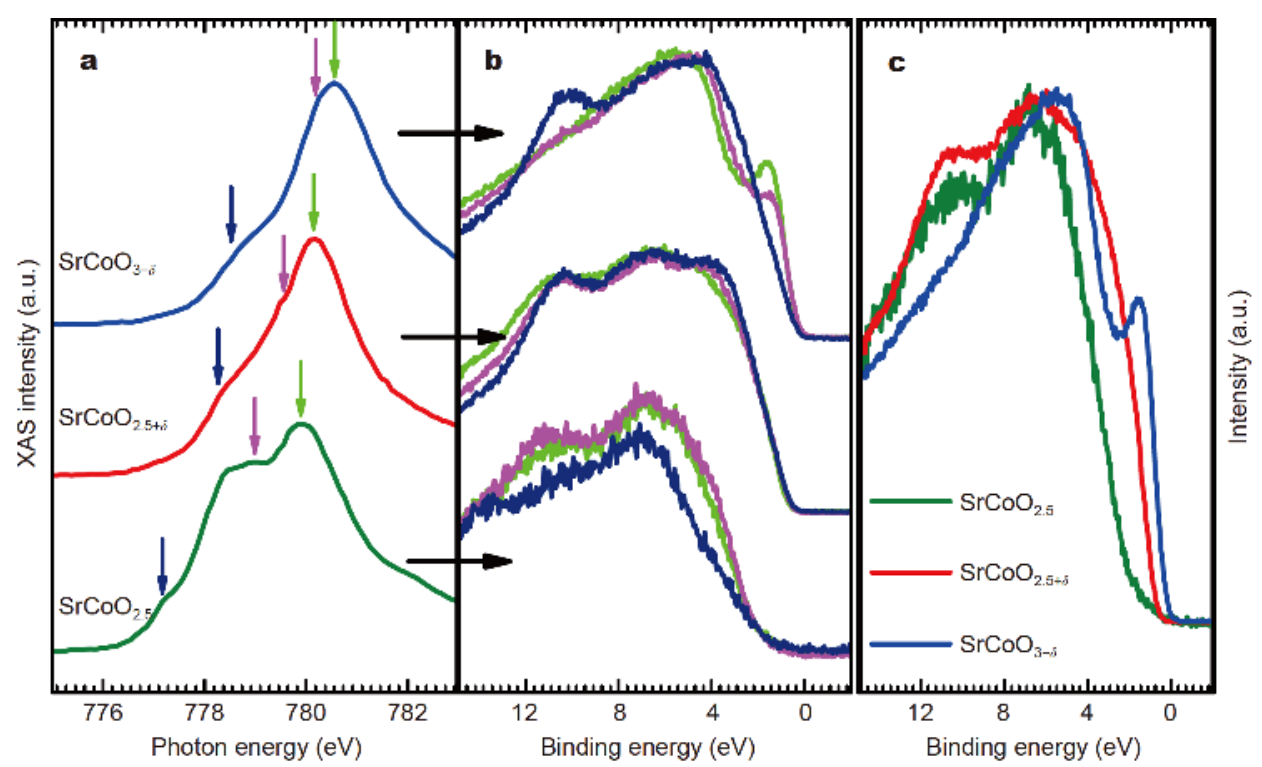

Figure 6 (a) Co- $\mathrm{L}_{3}$ edge XAS, (b) resonant photoemission spectra, (c) comparison of resonant photoemission of $\mathrm{SrCoO}_{3-x}$ thin films.

with the increase of oxygen content and results in the stronger hybridization between the Co $3 \mathrm{~d}$ and $\mathrm{O} 2 \mathrm{p}$ states, consistent with the results of the O-K edge XAS. For $\mathrm{SrCoO}_{3-\delta}$, the significant enhancement of peak intensity around $1 \mathrm{eV}$ below Fermi level at the on-resonance photon energy is observed. This peak has a Co $3 \mathrm{~d}$ characteristic, and the valence band maximum is dominated by the Co $3 \mathrm{~d}$ characteristic. The transformation from insulator $\left(\mathrm{SrCoO}_{2.5}\right)$ to metal $\left(\mathrm{SrCoO}_{3-\delta}\right)$ is also observed, as shown in Fig. 6c.

\section{CONCLUSIONS}

We have obtained three different oxygen-content $\mathrm{SrCoO}_{3-x}$ thin films by combing strain and chemical oxidation method: $\mathrm{SrCoO}_{2.5}, \mathrm{SrCoO}_{2.5+\delta}$, and $\mathrm{SrCoO}_{3-\delta}$. $\mathrm{XRD}$ and STEM-ABF not only demonstrate all films are of good crystalline quality and single phase, but also qualitatively determine the oxygen content of $\mathrm{SrCoO}_{3-x}$ thin films. The electronic structure evolution from an insulator $\mathrm{SrCoO}_{2.5}$ to a metal $\mathrm{SrCoO}_{3-\delta}$ was explored with XAS and RPES. As the increase of oxygen content, the hybridization between Co $3 \mathrm{~d}$ and $\mathrm{O} 2 \mathrm{p}$ is strengthened and the valence band maximum exhibits a typical $O 2 p$ characteristic. With further increasing oxygen content, $\mathrm{SrCoO}_{3-x}$ transform from a high spin $\mathrm{Co}^{3+}$ to an intermediate spin $\mathrm{Co}^{4+}$, resulting in a transition of insulator to metal. Our description of the electronic structure transition from insulator $\mathrm{SrCoO}_{2.5}$ to metal $\mathrm{SrCoO}_{3-\delta}$ is not only important for fundamental research, but also for industry applications, such as catalysts, solid oxide fuel cells, and so on.

Received 16 January 2019; accepted 28 February 2019; published online 22 March 2019

1 Lu Z, Wu X, Jiang M, et al. Transition metal oxides/hydroxides nanoarrays for aqueous electrochemical energy storage systems. Sci China Mater, 2014, 57: 59-69

$2 \mathrm{Lu} \mathrm{S}$, Zhuang Z. Electrocatalysts for hydrogen oxidation and evolution reactions. Sci China Mater, 2016, 59: 217-238

3 Kalinin SV, Spaldin NA. Functional ion defects in transition metal oxides. Science, 2013, 341: 858-859

4 Lan QQ, Zhang XJ, Shen X, et al. Tuning the magnetism of epitaxial cobalt oxide thin films by electron beam irradiation. Phys Rev Mater, 2017, 1: 024403

5 Xie CK, Nie YF, Wells BO, et al. Magnetic phase separation in $\mathrm{SrCoO}_{x}(2.5 \leq x \leq 3)$. Appl Phys Lett, 2011, 99: 052503

6 Choi WS, Jeen H, Lee JH, et al. Reversal of the lattice structure in $\mathrm{SrCoO}_{x}$ epitaxial thin films studied by real-time optical spectroscopy and first-principles calculations. Phys Rev Lett, 2013, 111: 097401

7 Jeen $\mathrm{H}$, Choi WS, Biegalski MD, et al. Reversible redox reactions in an epitaxially stabilized $\mathrm{SrCoO}_{x}$ oxygen sponge. Nat Mater, 2013, 12: $1057-1063$

8 Jeen H, Choi WS, Freeland JW, et al. Topotactic phase transformation of the brownmillerite $\mathrm{SrCoO}_{2.5}$ to the perovskite $\mathrm{SrCoO}_{3-\delta}$. Adv Mater, 2013, 25: 3651-3656

9 Lee JH, Choi WS, Jeen H, et al. Strongly coupled magnetic and electronic transitions in multivalent strontium cobaltites. Sci Rep, 2017, 7: 16066

10 Zhu ZH, Rueckert FJ, Budnick JI, et al. Distinct magnetic phases in structurally uniform $\mathrm{SrCoO}_{3-y}$. Phys Rev B, 2016, 93: 224412

11 Hu S, Seidel J. Oxygen content modulation by nanoscale chemical and electrical patterning in epitaxial $\mathrm{SrCoO}_{3-\delta}(0<\delta \leq 0.5)$ thin films. Nanotechnology, 2016, 27: 325301 
12 Zhang KHL, Sushko PV, Colby R, et al. Reversible nano-structuring of $\mathrm{SrCrO}_{3-\delta}$ through oxidation and reduction at low temperature. Nat Commun, 2014, 5: 4669

13 Lu N, Zhang P, Zhang Q, et al. Electric-field control of tri-state phase transformation with a selective dual-ion switch. Nature, 2017, 546: 124-128

$14 \mathrm{Lu} \mathrm{Q}$, Chen $\mathrm{Y}$, Bluhm $\mathrm{H}$, et al. Electronic structure evolution of $\mathrm{SrCoO}_{x}$ during electrochemically driven phase transition probed by in situ X-ray spectroscopy. J Phys Chem C, 2016, 120: 2414824157

15 Lim J, Yu J. Role of oxygen vacancy in the spin-state change and magnetic ordering in $\mathrm{SrCoO}_{3-\delta}$. Phys Rev B, 2018, 98: 085106

16 Zhao J, Guo H, He X, et al. Manipulating the structural and electronic properties of epitaxial $\mathrm{SrCoO}_{2.5}$ thin films by tuning the epitaxial strain. ACS Appl Mater Interfaces, 2018, 10: 10211-10219

17 Zhang Q, He X, Shi J, et al. Atomic-resolution imaging of electrically induced oxygen vacancy migration and phase transformation in $\mathrm{SrCoO}_{2.5-\sigma}$. Nat Commun, 2017, 8: 104

18 Long Y, Kaneko Y, Ishiwata S, et al. Synthesis of cubic $\mathrm{SrCoO}_{3}$ single crystal and its anisotropic magnetic and transport properties. J Phys-Condens Matter, 2011, 23: 245601

19 Lee JH, Rabe KM. Coupled magnetic-ferroelectric metal-insulator transition in epitaxially strained $\mathrm{SrCoO}_{3}$ from first principles. Phys Rev Lett, 2011, 107: 067601

20 Potze RH, Sawatzky GA, Abbate M. Possibility for an intermediate-spin ground state in the charge-transfer material $\mathrm{SrCoO}_{3}$. Phys Rev B, 1995, 51: 11501-11506

21 Zhuang $\mathrm{M}$, Zhang $\mathrm{W}, \mathrm{Hu} \mathrm{A}$, et al. Possible magnetic ground state in the perovskite $\mathrm{SrCoO}_{3}$. Phys Rev B, 1998, 57: 13655-13659

22 Karvonen L, Valkeapaa M, Liu RS, et al. O-K and Co-L XANES study on oxygen intercalation in perovskite $\mathrm{SrCoO}_{3-\delta}$. Chem Mater, 2010, 22: 70-76

Acknowledgements This work was supported by the National Key R\&D program of China (2016YFA0401002) and the National Natural Science Foundation of China (11574365, 11474349 and 11375228). The authors would like to thank BL14B1 beam line of Shanghai Synchrotron Radiation Facility for technique support.

Author contributions Zhao J designed and carried out most of the experiment, analyzed the results, and wrote the manuscript with support from Ibrahim K and Guo H. Luo Y helped to perform the experiment, including film growth and XRD measurements. Wang JO, Qian H and Liu C helped to perform the XAS and RPES measurements. Yang T and $\mathrm{Li} \mathrm{X}$ helped to perform the RSM measurements. Huang $\mathrm{H}$ performed the transport properties measurements. Gu L and Zhang Q contributed to STEM characterization. He M contributed to film growth. He X contributed to the theoretical analysis. Zhang B, Li S, Guo E, Ge C and Jin $\mathrm{KJ}$ revised the manuscript. All authors contributed to the general discussion.

Conflict of interest The authors declare no conflict of interest.

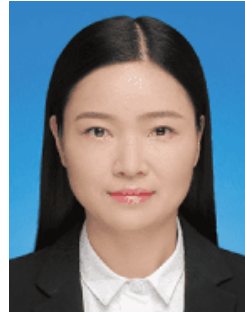

Jiali Zhao is a PhD candidate in the Institute of High Energy Physics, CAS. Her current research interest focuses on the electronic structure and physical properties of perovskite complex oxides films.

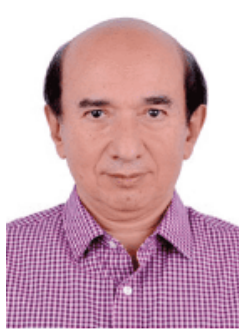

Kurash Ibrahim received his $\mathrm{PhD}$ degree in 1992 from the University of Liege, Belgium, worked as a postdoc fellow in the Institute of High Energy Physics, CAS (1993-1994), and has been a staff member since then. He worked as a visiting researcher in Lawrence Berkeley National Laboratory, USA (2002), KEK and HiSOR, Japan (2003); Department of Physics, University of Montana State, USA (2004-2006). His research interest is on the application synchrotron radiation to electronic structure investigation of condensed matter systems.

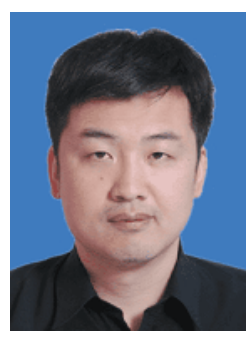

Haizhong Guo received his $\mathrm{PhD}$ degree in condensed matter physics from the Institute of Physics, CAS in 2005. From 2005 to 2009, he worked as a postdoc in the University of Alabama, University of California at Davis, and Louisiana State University. He had been an associate professor in the Institute of Physics, CAS (2009-2017). He joined Zhengzhou University as a professor in 2017. His research interest focuses on the novel physical phenomena emerging in the low-dimension transition-metal oxide heterostructures.

\section{$\mathrm{SrCoO}_{3-x}$ 薄膜中氧空位引起的电子结构演变}

赵佳丽 ${ }^{1,5}$, 罗毅 ${ }^{2}$, 王嘉鸥 ${ }^{1}$, 钱海杰 ${ }^{1}$, 刘晨 ${ }^{1}$, 何旭 ${ }^{3}$, 张庆华 ${ }^{4,5}$, 黄河意 ${ }^{4,5}$, 张兵兵 ${ }^{1}$, 李顺芳 ${ }^{2}$, 郭尔佳 ${ }^{4}$, 葛琛 ${ }^{4}$, 杨铁莹 ${ }^{6}$, 李晓龙 ${ }^{6}$, 何萌 ${ }^{4}$, 谷林 ${ }^{4,5}$, 金奎娟 ${ }^{4,5}$, 奎热西・依布拉欣 ${ }^{*}$, 郭海中 ${ }^{2^{*}}$

摘要 离子缺陷, 比如氧空位, 在过渡金属氧化物的磁性和电子结 构中起着至关重要的作用. 对氧空位进行调控广泛应用于催化和 能量转换领域. 我们研究了 $\mathrm{SrCoO}_{3-x}$ 的电子结构与氧含量 $x$ 的关系, 发现随着氧空位含量的减少, Co $3 \mathrm{~d}$ 和 $\mathrm{O} 2 \mathrm{p}$ 之间的杂化增强. $\mathrm{SrCoO}_{2.5+\delta}$ 的价带顶具有明显的 $\mathrm{O} 2 \mathrm{p}$ 特征. 随着氧含量的进一步增 加, $\mathrm{Co}$ 离子从高自旋的 $\mathrm{Co}^{3+}$ 变成了中自旋的 $\mathrm{Co}^{4+}$, 实现了绝缘体到 金属的转变. 关于 $\mathrm{SrCoO}_{3-x}$ 中氧空位引起电子结构演变的研究不仅 展示了Co离子自旋的转变, 同时也为其在催化和能源领域的应用 提供了理论支持. 